\title{
Women with breast cancer's perceptions of nurse-led telephone-based motivational interviewing consultations to enhance adherence to adjuvant endocrine therapy: a qualitative study
}

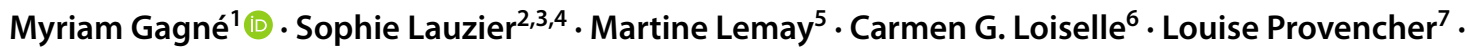 \\ Chantale Simard ${ }^{8} \cdot$ Laurence Guillaumie $^{2,9,10}$
}

Received: 26 May 2021 / Accepted: 10 November 2021 / Published online: 8 February 2022

(c) The Author(s), under exclusive licence to Springer-Verlag GmbH Germany, part of Springer Nature 2022

\begin{abstract}
Background Daily adjuvant endocrine therapy (AET) for 5 or 10 years is the standard of care for women diagnosed with non-metastatic hormone receptor-positive breast cancer. However, many women experience AET-related issues that may hamper quality of life and adherence. Here, we aimed to describe women's perceptions of motivational interviewing (MI)guided consultations delivered by a trained nurse navigator over the telephone to enhance AET adherence.

Methods Eighteen women who were first prescribed AET for non-metastatic breast cancer in the last 5 years, who selfreported AET-related issues, and who participated in at least two MI-guided consultations over a year were interviewed about their perceptions of the intervention, using a semi-structured interview guide. Audio recordings were transcribed verbatim and analyzed using a thematic analysis approach.

Results Three main themes emerged from the data about women's perceptions on MI-guided consultations. These consultations were described as (1) a person-centred experience, (2) providing key information about AET, and (3) supportive of present and future AET experience, by contributing to AET side-effect management, motivation, adherence, calming negative emotions, improving well-being and self-esteem, and making women to feel empowered.

Conclusions Nurse-led telephone-based MI-guided consultations about AET were found to respond to participants' needs and to enhance participants' perceptions of being informed and being supported in experiencing various facets of AET. Telephone-based consultations for AET are perceived as a promising strategy in an increasing virtual care world.
\end{abstract}

Keywords Breast neoplasms · Motivational interviewing · Oncology nursing · Qualitative research · Medication adherence

\section{Abbreviations}

AET Adjuvant endocrine therapy

MI Motivational interviewing

\section{Background}

Breast cancer is the most common cancer in women, with a global incidence of $>2$ million incident cases worldwide in 2018 [1,2]. Over the last three decades, there has been a substantial decline in breast cancer mortality [3], mainly attributed to early detection [4], timely surgery, and treatment, including adjuvant endocrine therapy (AET) [5].

Laurence Guillaumie

Laurence.Guillaumie@fsi.ulaval.ca

Extended author information available on the last page of the article
Generally taken after the completion of other treatments (e.g. surgery, chemotherapy, radiotherapy), daily AET tamoxifen or aromatase inhibitors - for 5 or 10 years is now the standard of care for women diagnosed with nonmetastatic hormone receptor-positive breast cancer [6] (approximately 60-75\% of all breast cancer diagnoses) [7]. AET lowers the risk of breast cancer recurrence among women by $47 \%$ over 5 years and reduces the risk of breast cancer mortality by $30 \%$ over 15 years [8]. Despite these benefits, suboptimal AET implementation and treatment discontinuation are common among women prescribed AET and are associated with increased healthcare costs and risk of mortality [9].

AET side-effects have been shown to hamper quality of life [10] and adherence [11]. Side-effects vary according to the type of AET and may include vasomotor, musculoskeletal, neuropsychiatric, gastrointestinal, and gynecological symptoms [12]. In addition to the frequency and intensity 
of AET side-effects $[11,13,14]$, other potentially modifiable factors are associated with AET non-adherence. These include lack of patient involvement in treatmentrelated decision-making [15]; lack of information about the pros and cons of AET (including side-effects) at the time of being prescribed [13-16]; lack of support during treatment [15], including lack of support with coping with side-effects [16, 17]; and negative perceptions of AET [13, $17,18]$. In contrast, women who show better adherence to AET are those who are given the opportunity to ask questions about AET before initiating treatment [14]; clearly understand treatment outcomes, including side effects [14]; have access to continued follow-up care [17]; are satisfied with their patient-provider relationship [16, 17]; and feel supported by the cancer care team [13, 16].

To date, few interventions to promote adherence to AET in breast cancer women have been evaluated, and those that were shown little or no effect [19]. However, interventions in which AET was discussed between patients and providers resulted in significant improvements in AET adherence, compared to usual care [19]. Motivational interviewing (MI) is a theoretically based and personcentred approach that guides open patient-provider discussions about self-management to enhance patient motivation and drive behaviour change [20]. MI has been shown to drive health behaviours such as physical exercise among individuals with cancer [21] and enhance adherence to pharmacological treatments among patients with chronic diseases [22, 23].

Based on a previous need assessment study which identified side-effects as a major barrier to taking AET [16], two research team members (SL, LG), in collaboration with oncology nurses, developed an MI guide for AET counselling (Table 1). This guide formed the basis of an MI-guided intervention, whereby oncology nurse navigators were trained to provide women with a minimum of two 15-min consultations guided by an MI approach over the telephone. This 1-year intervention was supported by evidence-based pharmacological and non-pharmacological strategies to cope with 19 side-effects and other AETrelated concerns (additional details provided in Supplementary Material 1).

Although MI consultations are often led by nurses and conducted either partially or entirely over the telephone [21,23], no previous study has sought to gather women with breast cancer views of MI for AET. As a first step toward assessing the perceived impacts of such novel intervention, we aimed to explore participants' perceptions pertaining to these telephone-based and MI-guided consultations provided by a trained oncology nurse navigator in women who were prescribed AET for non-metastatic breast cancer and who reported AET-related issues.

\section{Methods}

\section{Design}

We conducted a descriptive qualitative study among women with breast cancer who participated in this MI-guided intervention and used a computer-assisted thematic content analysis approach [24]. The present study was approved by CHU de Québec-Université Laval Research Ethics Board (approval number: MR-20-2018-3654).

\section{Participants and recruitment procedures}

Eligibility criteria for participants included the following: being $\geq 18$ years of age, having received an initial diagnosis of non-metastatic hormone-sensitive breast cancer at the CHU de Québec-Université Laval (a tertiary care centre in Québec City, Québec Canada), having received a first prescription for AET in the last 5 years, self-reporting AETrelated issues, and being able to converse fluently in French. Participants were not eligible if they were diagnosed with a severe mental illness (as documented in their medical chart), had hearing impairment that would compromise their participation in telephone consultations, were already enrolled in a study on AET, or were required by their physician to discontinue AET. Finally, we excluded women who did not attend at least two MI-guided consultations.

Recruitment materials were posted at the Centre des maladies du sein. Healthcare professionals involved in cancer care could also invite women to participate in the study. Potentially eligible women who were interested in the study were invited to contact the study coordinator by telephone to assess eligibility. All participants provided verbal informed consent (audio recorded) and completed a sociodemographic and background questionnaire at study entry.

\section{Data collection}

Within the month that followed the last MI-guided consultation, each participant was booked for an in-person or telephone semi-structured interview with a research assistant (interview guide in Supplementary Material 2). The interview covered topics such as participant's subjective description of telephone-based MI-guided consultations, perceived impact and overall satisfaction with the program, and areas for improvement.

\section{Data analysis}

Interviews were audio-taped and transcribed verbatim. Transcripts were checked to ensure they did not contained 
Table 1 MI-guided consultation guide

\begin{tabular}{|c|c|}
\hline Consultation stage & Sample questions and opening sentences \\
\hline \multicolumn{2}{|l|}{ 1. Initial engagement } \\
\hline Introduce yourself & Hello, [person's name]. My name is [nurse's name] and I am an oncology nurse. \\
\hline Express interest and availability & $\begin{array}{l}\text { Please ask me any questions you might have and don't hesitate to interrupt me. The goal } \\
\text { of this exchange is to best meet your needs. }\end{array}$ \\
\hline \multicolumn{2}{|l|}{ 2. Focus } \\
\hline Inquire about concerns and difficulties & $\begin{array}{l}\text { How is your treatment going? } \\
\text { Do you have any other concerns? }\end{array}$ \\
\hline Explore what has already been tried & Have you tried anything to improve the situation? \\
\hline Inquiring about treatment adherence & $\begin{array}{l}\text { It can be hard to remember to take medication every day without forgetting. How is that } \\
\text { going for you? Is there anything you've tried to help you remember to take it? }\end{array}$ \\
\hline Prioritizing and agreeing on topics & What's bothering you the most? Let's examine that first. \\
\hline \multicolumn{2}{|l|}{ 3. Raising the issue and planning strategies } \\
\hline Clarifying struggles & You told me about [struggle]. Can you tell me a bit more about that? \\
\hline Informing & I could tell you about some strategies for this side-effect/situation \\
\hline Invite the person to choose strategies & Is there anything else you'd like to try? \\
\hline If needed, provide information & Regarding [strategy mentioned], it's good to know that [give information] \\
\hline If necessary, invite them to discuss other strategies & Are there any other strategies you'd like to try? \\
\hline If they mention several strategies, go over them & Could you go over what you'd like to try? \\
\hline \multicolumn{2}{|l|}{ a. Addressing low motivation } \\
\hline Naming the situation & You've already tried a lot of options without improvement \\
\hline Recall treatment benefits & $\begin{array}{l}\text { On the one hand, treatment has its difficulties; on the other hand, there are benefits. } \\
\text { What have you been told about treatment benefits? }\end{array}$ \\
\hline Support their choice and experience & It's understandable that you would want to preserve your quality of life. \\
\hline If stopping treatment, inform the doctor & If it's okay with you, I'll let your doctor know. \\
\hline If stopping treatment, emphasize availability & Don't hesitate to call us if you have any questions or if you'd like to discuss this further. \\
\hline 4. Conclusion & Sample questions and opening sentences \\
\hline Check if they need further information & Do you have all the information that you need? \\
\hline Provide encouragement & I'm confident that, with all of this, your situation will improve. \\
\hline Recall treatment benefits & $\begin{array}{l}\text { The take-home message is the benefits of treatment compared to the disease. } \\
\text { You have to take your medication every day, for at least } 5 \text { or } 10 \text { years, as recommended } \\
\text { by your doctor. }\end{array}$ \\
\hline Arrange a follow-up call & Can I give you a call back in $[\mathrm{X}]$ weeks/months? \\
\hline Remind them of your availability & Don't hesitate to call us if you have any questions between now and then. \\
\hline
\end{tabular}

mistakes. De-identified transcripts were imported to QSR NVivo 11 software ${ }$. A trained qualitative data analyst read and coded transcripts line by line, and developed the codebook following a process inspired by a thematic analysis approach, whereby codes are derived both from the literature and the topic guide, and from the corpus itself [24]. Memos were used throughout the process, and codes were crosschecked by our lead researcher (LG). Codes were sorted into categories and categories into themes, through converging perspectives from participants. Emerging themes and categories were discussed among three researchers (LG, SL, and MG) and adjusted for consensus. To capture a variety of perceptions of telephone-based MI-guided consultations on AET, we initially planned on recruiting at least 20 participants, but interviews were conducted until data saturation was reached.

\section{Results}

A total of 30 women contacted the research team to obtain information about the study and 23 agreed to participate (of the 30, 5 women were non-eligible, 1 could not be reached, and 1 declined to participate). Two women had attended a single consultation over the study period and were excluded. Twenty-one women had between 2 and 5 consultations (mean consultation length: $23 \mathrm{~min}$ ). The first three interviews with women were used to refine the initial interview guide. A final sample of 18 women completed all study requirements and were included in the present dataset.

Sociodemographic and background characteristics are presented in Table 2. Participants were 55 to 78 years of age (mean: 65 years), about half had a university degree $(n=$ 8 ) and fourteen had a prescription for an aromatase inhibitor. 
Table 2 Characteristics of participants at study entry ( $n$ $=18$ )

$$
\text { Characteristics }
$$

Mean (minimum to maximum) or $n$

Age

Highest attained level of education

High school

College diploma

Partial university degree

University degree

Annual family income

CA $\$ 30,000.00$ to CA $\$ 39,999.99$

CA $\$ 40,000.00$ to CA $\$ 59,999.99$

CA $\$ 60,000.00$ to CA $\$ 79,999.99$

CA $\$ 80,000.00$ to CA $\$ 99,999.99$

Does not know

Comorbid physical or mental health problem(s)

$$
\text { Yes }
$$

No

Time since breast cancer diagnosis

Cancer treatment(s) received

Breast surgery

Axillary surgery

Chemotherapy

Radiotherapy/brachytherapy

Trastuzumab

Previous AET

Yes

No

Time since first prescription of AET

AET at study entry

Tamoxifen

Letrozole

Anastrozole

Exemestane

Self-reported side-effect(s) of AET at study entry

Vasomotor symptoms

$\begin{array}{ll}\text { Hot flashes } & 14 \\ \text { Cold sweats } & 7 \\ \text { Night sweats } & 11\end{array}$

Gynecological symptoms

Vaginal discharge

Vaginal itching/irritation

Vaginal dryness

Pain or discomfort with intercourse

Lost interest in sex

Breast sensitivity/tenderness

Gastrointestinal symptoms

Weight gain

Diarrhea

Bloated feeling

Musculoskeletal symptoms

Arthralgia

Neuropsychiatric symptoms

Headaches
65 years (from 55 to 78 years)

2

7

1

8

3

7

3

2

3

13

5

22 months (from 3 to 43 months)

17

14

7

15

2

7

11

14 months (from 0 to 36 months)

4

6

7

1

14

6

11

4

11

9

4

5

6

18

9 
Table 2 (continued)

\begin{tabular}{ll}
\hline Characteristics & Mean (minimum to maximum) or $n$ \\
\hline Lightheaded (dizzy) feeling & 7 \\
Mood swings & 6 \\
Irritability & 9 \\
\hline
\end{tabular}

$A E T$ adjuvant endocrine therapy, $C A \$$ Canadian dollar

Participants reported experiencing a variety of AET sideeffects, and these side-effects were their main motivation to take part in the study.

\section{Women's perceptions on telephone-based motivational interviewing (MI)-guided consultations delivered by a trained nurse navigator}

Three main themes emerged from the analysis (Table 3). These are described below.

\section{MI-guided consultations perceived as a person-centred experience of care}

Participants expressed that MI-guided consultations provided by a nurse navigator focused on their experience with and concerns about AET. Throughout the intervention, participants reported feeling important and actively supported by their nurse navigator. Participants also revealed that they felt free to express and discuss what mattered the most to them during these consultations. As such, MI-based consultations contrasted with previous experiences of cancer care, where, at times, barriers to open communication were faced.

The consultation was really about me, about what I was going through. It was about the things that I was most concerned about [...]. (P025).

I didn't feel like the nurse was in a hurry. She took all the time needed to answer my questions. One time, I told her about my arthritic pain in my ankles. She listened to me, gave me some advice. During the next call, she took the trouble to find out what I had done and what the outcome was. (P027).

In the doctor's office, it's less easy [than with the nurse navigator] to talk about very personal things. Doctors don't always have time to listen to you. Talking to a pharmacist about intimate problems is even more embarrassing! (P022).

Table 3 Main findings related to breast cancer women's perceptions of MI-guided consultations on AET provided by a trained nurse navigator over the phone

MI-based consultations are described as:

1. A person-centred experience:

1.1 Focussing on breast cancer women's personal concerns about AET;

1.2. Making breast cancer women to feel important;

1.3 Making breast cancer women to feel supported;

1.4 Making breast cancer women to feel free to discuss what mattered the most to them;

1.5 Contrasting with previous experiences of cancer care

2. Providing key information for AET self-management:

2.1. With nurse navigators demonstrating a high level of knowledge about how to cope with side-effects of AET;

2.2 With nurse navigators seeking additional information, where needed;

2.3 With nurse navigators suggesting breast cancer women to seek advice from other healthcare professionals to alleviate side-effects of AET;

2.4 With nurse navigators demonstrating a high level of competence in managing AET-related issues;

3. Supportive of present and future AET experiences

3.1 Sometimes contributing to alleviate side-effects of AET, sometimes not;

3.2 Sometimes strengthening motivation to take AET, sometimes not;

3.3 Sometimes improving adherence to AET, sometimes not;

3.4 Calming negative emotions related to AET side-effects, including anxiety;

3.5 Improving breast cancer women's well-being and self-esteem;

3.6 Making breast cancer women to feel empowered.

$A E T$ adjuvant endocrine therapy 


\section{MI-guided consultations perceived as providing key information for self-management}

Nurse navigators were reported to provide participants with key evidence-based information to alleviate AET-related issues. Specifically, participants described nurse navigators as demonstrating a high level of knowledge of AET sideeffect management. When nurse navigators were unable to give advice to mitigate AET-related issues during a particular phone call, they were appraised for seeking additional information. Participants also reported that nurse navigators suggested they solicit advice from other healthcare professionals (e.g.: sexologist, kinesiologist) to overcome AETrelated issues reported during consultations. As such, participants gratefully acknowledged nurse navigators for their continuing efforts and high competence level in managing side-effects of AET.

I learned more about the medication [with the nurse navigator] than when it was prescribed for me. (P008). The information the nurse navigator gave me made a huge difference. Talking to her about my vaginal atrophy problems led me to see a sexologist and a physiotherapist. I didn't even know there was such a thing as perineal rehabilitation [...]. (P006).

\section{MI-guided consultations perceived as supportive of present and future adjuvant endocrine therapy (AET) experiences}

For some participants, implementing changes suggested by nurse navigators successfully reduced side-effects of AET. For others, adopting suggestions did not resolve these issues. Other women were unable or not ready to follow recommendations formulated by the nurse navigator.

The strategies the nurse suggested to reduce sideeffects helped me. (P023)

The nurse made some very relevant suggestions. The problem is, I already knew them and they didn't help. (P021)

Some participants also reported that discussions with the nurse navigator strengthened their motivation to continue to take AET. Those who reported being highly motivated to adhere to their prescribed AET at baseline also expressed that taking part in these consultations did not further improve their motivation. These participants, however, reported that taking part in the intervention was mainly driven by their learning needs to improve self-management, particularly related to side-effects.

At first, I was very motivated to take adjuvant hormone therapy. What prompted me to participate in the program was to find solutions to reduce the side-effects. [...]. (P023)
I don't need to be motivated: I'm taking the medication because I want to avoid a recurrence of cancer in my other breast. (P014)

Although efforts made by nurse navigators to help participants to alleviate side-effects were sometimes unsuccessful, several women reported that taking part in telephone-based MI-guided consultations improved their level of adherence to AET, either initiation, daily intake, or persistence for the recommended duration.

Before I talked to the nurse, there was no way I was going to take adjuvant hormone therapy. I wasn't convinced at all. [...] I tried it and now taking the medication is part of my routine. (P011)

It was after I talked to the nurse that I took my medication all the time $[\ldots](\mathrm{P} 010)$.

Being part of the program led me to continue taking the medication. (P029)

Although the perceived benefits of attending MI-guided consultations varied among participants in terms of motivation, side-effect management, and AET adherence, participants expressed that taking part in the intervention appeared to play a crucial role in reducing negative emotions related to AET-related issues. Some participants reported that MIguided consultations reduced their anxiety about side-effects of AET. Others who could not alleviate AET side-effects despite implementing suggestions provided by the nurse navigator nevertheless reported that being well informed on AET benefits and available strategies for side-effect management helped them to persist with the treatment.

Participating in the program made me realize that my situation wasn't permanent, that I could find ways to overcome it and not be afraid. (P013)

I was really fed up. I found it extremely difficult. I still find it hard, but I console myself by telling myself that I have the answers to my questions. (P025)

As for other participants, MI-guided consultations were reported to elicit positive outcomes such as improved wellbeing, self-esteem, and empowerment.

Participating in the program made me feel like someone was taking care of me. I had someone I could ask questions, someone who would call me, talk to me in their gentle and comforting voice, and who was nice to me. (P019)

I felt valued. Participating in the program gave me a lot of self-esteem: not to let things slide, not to give up, even if I'm taking a medication and I have sideeffects. (P006)

It changed my well-being a lot. I have less pain, less heat. I' $m$ in better spirits. I' $m$ more active. I go out more. I see more people. I'm less anxious. (P022) 


\section{Convenience of telephone consultations}

Participants appreciated having access to a timely support from a nurse navigator over the telephone. Interviews also revealed that telephone consultations enabled helpful exchanges in women who lived in rural areas or in those with limited transport options to reach the hospital. Others, however, reported that they would have preferred in-person consultations.

I enjoyed being able to have direct access to the nurse without having to go through the reception desk, make an appointment by telephone. I could just call her and tell her that I wasn't feeling well, that I had such and such a side-effect. (P029)

I prefer to see the people I' $m$ talking to. [...] I find it easier face-to-face. (P010)

\section{Areas for improvement}

When questioned about how the current program could be improved, some women recommended providing telephonebased MI-guided consultations soon after AET prescription, to every woman prescribed this therapy. Others suggested extending the program beyond the 12-month period. Some participants also proposed that pharmacists would be well positioned to offer MI-guided consultations.

\section{Discussion}

\section{Key findings}

This qualitative study explored breast cancer women's perceptions pertaining to telephone-based nurse-led MI-guided consultations to improve adherence with AET. For many, taking part in these consultations alleviated side-effects, inspired optimism for long-term use of AET, and strengthened intrinsic motivation to initiate, implement, or persist with the treatment, based on a true understanding of the benefits of taking AET for the prescribed duration and how to cope with side-effects. The positive aspects of these MIguided consultations are further discussed below.

First, our findings show that the MI-guided intervention is perceived as affecting participants' experience with managing side-effects of AET, a major AET-related issue [12], and the main reason for developing this phone-support program [16]. Throughout the intervention, study participants were provided with tailored, evidence-based information on available strategies to mitigate AET side-effects and invited to co-develop their self-management plan. Whether or not the agreed upon strategies succeeded in alleviating
AET-related issues, women reported feeling engaged in their care, supported by attentive nurse navigators, and expressed being more informed about side-effects of AET. Considering the paucity of studies on MI for side-effect management [25-27], these findings extend current knowledge, underlining the role of core MI steps in satisfying the educational [13-16] needs of non-metastatic breast cancer patients, as well as their desire to be actively involved in their care [15] and supported by their providers [15-17].

Second, our findings show that MI-guided consultations are perceived as exerting influence on participants' perceptions of AET, whereby provision of tailored evidence-based information increases women's sense of empowerment and optimism. Previous literature has shown that side-effects and fear of long-term consequences of AET are associated with lower adherence [17]. By informing women that several evidence-based options are available to mitigate AET-related issues, by encouraging them to implement a preference-based strategy and following-up on its efficacy, and by providing women with ongoing and timely support, the current MI-guided intervention contributed to building optimism for taking AET for several years, even in the case where difficulties were not solved. Considering the long and arduous breast cancer journey and the intangibility of AET benefits, promoting a positive and hopeful view of the long-term AET intake appears as valuable contribution of MI-guided consultations to breast cancer women. Consistent with our findings, nurse navigators involved in addressing addictions in cancer patients reported that MI techniques contributed to increasing patients' empowerment [28]. A tailored physical activity program supported by providers trained in MI techniques also fuelled sense of optimism for the future in people with mental health conditions [29]. Among patients with diabetes, combining information provision, MI techniques, and meaningful relationships led to improved sense of control [30], while others reported that it drove optimism [31]. Provision of information combined with MI techniques was also identified as two intervention components perceived as most beneficial in people receiving antiretroviral therapy [32]. Consistent with this, our study shows that the availability of tailored, accurate, up-to-date, and complete information on how to cope with AET-related issues as well as breast cancer women's involvement in care, coupled with the ongoing, timely support from nurse navigators, created synergies that were perceived to grow women's empowerment and optimism for the long-term intake of AET.

Third, in accordance with MI theory [33], our findings show that MI-guided consultations on AET are perceived as wielding influence over women's motivation to persist with AET. The intervention allowed to reinforcing intrinsic motivation in women who were highly motivated to take long-term AET but who faced AET-related issues at study 
entry, and shifting from extrinsic to intrinsic motivation in women who encountered AET-related difficulties and who lacked personal reasons for adhering to it. In line with these results, previous studies demonstrated (with the lens of Self-Determination Theory [34]) the contributions of MI for shifting from extrinsic to intrinsic motivation [35, 36]. In cancer survivors, these psychological needs have been shown to ascribe positive meaning during survivorship and lead to improved social well-being [37]. Similarly, through MI-guided AET counselling, our study indicates that participants perceive acquiring knowledge about AET, improving perception of control, and building meaningful relationship with their nurse navigator. In addition, women who might have first considered taking AET on their doctors' recommendation only (extrinsic motivation) may have finally chosen to initiate or persist with the AET to increase their own chance of avoiding recurrence and increasing survival (intrinsic motivation), as they became gradually aware of the role, benefits, and strategies to deal with AET-related issues and adopted a more positive view of the long-term AET intake. This finding is consistent with emerging literature in chronic disease management, whereby MI is shown to strengthen treatment motivation through increasing satisfaction with life despite undesirable side-effects [38] and enhance persistence with long-term medications [39, 40].

\section{Strengths and limitations}

This study bridges important knowledge gaps, as it is the first (to the best of our knowledge) to describe non-metastatic breast cancer women's perceptions of telephone-based MI-guided consultations on AET. Our qualitative approach enabled us to explore the potential benefits of our intervention, along with the process by which these benefits may be achieved. Our findings pertain to women who reported difficulties with AET.

Although the intervention was inspired by MI principles, it did not entirely espouse this approach [33]. However, implementing MI has been perceived as complex by providers, and this perception has acted as a major barrier MI uptake in real practice [41]. Major strengths of the current consultations therefore lie in that the intervention was designed to meet pre-assessed women's needs in regards to AET [16], inspired by core MI principles while being codeveloped with experienced oncology nurse navigators and thereby meeting specific requirements for real-world cancer care. Although women suggested to deliver the intervention sooner after the receiving their prescription, the best timing to implement it should be further explored and is likely to depend on women's motivation level, which varies at the time of initiating the treatment and/or over time, as a result of side-effects. In this context, another important strength to acknowledge is the flexibility of the present intervention, which can be easily adapted to meet participants' needs in terms of intensity, frequency, duration, and individual selfmanagement objectives.

\section{Conclusions}

This study suggests that non-metastatic breast cancer women struggling with AET-related issues perceive a variety of benefits related to attending between two and five nurse-led telephone-based MI-guided consultations about long-term AET intake. Our findings support a further quantitative assessment of this approach, especially in women with low intrinsic motivation at the time of initiating the treatment or in those in whom motivation is negatively reduced by side-effects, and call for expanding common success metrics of MI consultations for breast cancer women. Also, 1 year into the COVID-19 pandemic, healthcare has largely shifted from in-office visits to virtual care, including in breast cancer [42]. Hence, this study adds to a growing body of evidence that virtual care, including phone calls delivered by allied health professionals, could be used to improve survivorship outcomes in cancer [43, 44].

Supplementary Information The online version contains supplementary material available at https://doi.org/10.1007/s00520-021-06692-x.

Acknowledgements The authors thank Amel Baghdadi, MSc; Véronique Turcotte, MSc; Laurence Lambert-Côté, MSc; and Mauranne Labonté, MSc, for coordinating the study. The authors are grateful to Pierre-Olivier Lamarche, MA, for performing qualitative data analysis, and to Barbara Pattison, CTr, for translating quotations from French to English. The authors wish to thank all breast cancer patients and oncology nurse navigators who kindly volunteered their time to participate in the present study.

Author contribution SL, ML, CGL, LP, CS, and LG designed the study. LP was involved in participant recruitment. SL and LG contributed to conducting qualitative analyses. All authors contributed to data interpretation. MG, SL, and LG wrote the initial draft, and all other authors revised it for important intellectual content. All authors gave final approval of the version to be published. LG has full access to the data and take responsibility for the integrity of the data and accuracy of the data analysis.

Funding The Réseau de recherche en interventions en sciences infirmières du Québec (Québec Network on Nursing Intervention Research) funded the study. MG is a postdoctoral fellow supported by the Canadian Institutes for Health Research and the Fonds de recherche $d u$ Québec-Santé (Québec Health Research Fund). SL is research scholar with funding from the Québec Health Research Fund. CGL's research is supported by the Christine and Herschel Victor/Hope and Cope Chair in Psychosocial Oncology.

Availability of data Please contact the corresponding authors to access the study data.

Code availability Not applicable 


\section{Declarations}

Ethics approval The study has been approved by the $C H U$ de QuébecUniversité Laval Ethics Committee (approval number: MR-20-20183654).

Consent to participate Informed consent was obtained from all individual participants included in the study.

Consent for publication All participants agreed with publication of de-identified quotations.

Conflict of interest The authors declare no competing interests.

\section{References}

1. Heer E, Harper A, Escandor N, Sung H, McCormack V, FidlerBenaoudia MM (2020) Global burden and trends in premenopausal and postmenopausal breast cancer: a population-based study. Lancet Glob Health 8:e1027-e1037

2. Li N, Deng Y, Zhou L, Tian T, Yang S, Wu Y et al (2019) Global burden of breast cancer and attributable risk factors in 195 countries and territories, from 1990 to 2017: results from the Global Burden of Disease Study 2017. J Hematol Oncol 12:140

3. Brenner DR, Weir HK, Demers AA, Ellison LF, Louzado C, Shaw A et al (2020) Projected estimates of cancer in Canada in 2020. CMAJ 192:E199-E205

4. Oeffinger KC, Fontham ET, Etzioni R, Herzig A, Michaelson JS, Shih YC et al (2015) Breast cancer screening for women at average risk: 2015 guideline update from the American Cancer Society. JAMA 314:1599-1614

5. Edwards BK, Brown ML, Wingo PA, Howe HL, Ward E, Ries LA et al (2005) Annual report to the nation on the status of cancer, 1975-2002, featuring population-based trends in cancer treatment. J Natl Cancer Inst 97:1407-1427

6. Burstein HJ, Lacchetti C, Anderson H, Buchholz TA, Davidson NE, Gelmon KA et al (2019) Adjuvant endocrine therapy for women with hormone receptor-positive breast cancer: ASCO clinical practice guideline focused update. J Clin Oncol 37:423-438

7. Anderson WF, Chatterjee N, Ershler WB, Brawley OW (2002) Estrogen receptor breast cancer phenotypes in the Surveillance, Epidemiology, and End Results database. Breast Cancer Res Treat 76:27-36

8. Early Breast Cancer Trialists Collaborative Group (2011) Relevance of breast cancer hormone receptors and other factors to the efficacy of adjuvant tamoxifen: patient-level meta-analysis of randomised trials. Lancet 378:771-784

9. Hershman DL, Shao T, Kushi LH, Buono D, Tsai WY, Fehrenbacher L et al (2011) Early discontinuation and non-adherence to adjuvant hormonal therapy are associated with increased mortality in women with breast cancer. Breast Cancer Res Treat 126:529-537

10. Davis LE, Bubis LD, Mahar AL, Li Q, Sussman J, Moody L et al (2018) Patient-reported symptoms after breast cancer diagnosis and treatment: a retrospective cohort study. Eur J Cancer 101:1-11

11. Blok EJ, Kroep JR, Meershoek-Klein Kranenbarg E, Duijm-de Carpentier M, Putter H, Liefers GJ et al (2018) Treatment decisions and the impact of adverse events before and during extended endocrine therapy in postmenopausal early breast cancer. Eur $\mathbf{J}$ Cancer 95:59-67

12. Cella D, Fallowfield L, Barker P, Cuzick J, Locker G, Howell A et al (2006) Quality of life of postmenopausal women in the ATAC ("Arimidex", Tamoxifen, alone or in combination) trial after completion of 5 years' adjuvant treatment for early breast cancer. Breast Cancer Res Treat 100:273-284

13. Cahir C, Guinan E, Dombrowski SU, Sharp L, Bennett K (2015) Identifying the determinants of adjuvant hormonal therapy medication taking behaviour in women with stages I-III breast cancer: a systematic review and meta-analysis. Patient Educ Couns 98:1524-1539

14. Cluze C, Rey D, Huiart L, BenDiane MK, Bouhnik AD, Berenger $C$ et al (2012) Adjuvant endocrine therapy with tamoxifen in young women with breast cancer: determinants of interruptions vary over time. Ann Oncol 23:882-890

15. Kahn KL, Schneider EC, Malin JL, Adams JL, Epstein AM (2007) Patient centered experiences in breast cancer: predicting long-term adherence to tamoxifen use. Med Care 45:431-439

16. Humphries B, Collins S, Guillaumie L, Lemieux J, Dionne A, Provencher L et al (2018) Women's beliefs on early adherence to adjuvant endocrine therapy for breast cancer: a theory-based qualitative study to guide the development of community pharmacist interventions. Pharmacy 6(2):53. https://doi.org/10. 3390/pharmacy6020053

17. Lambert LK, Balneaves LG, Howard AF, Chia SK, Gotay CC (2018) Understanding adjuvant endocrine therapy persistence in breast Cancer survivors. BMC Cancer 18:732

18. Brier MJ, Chambless DL, Chen J, Mao JJ (2018) Ageing perceptions and non-adherence to aromatase inhibitors among breast cancer survivors. Eur J Cancer 91:145-152

19. Finitsis DJ, Vose BA, Mahalak JG, Salner AL (2019) Interventions to promote adherence to endocrine therapy among breast cancer survivors: a meta-analysis. Psychooncology 28:255-263

20. Miller WR (1983) Motivational Interviewing with problem drinkers. Behav Psychother 11:147-172

21. Spencer JC, Wheeler SB (2016) A systematic review of Motivational Interviewing interventions in cancer patients and survivors. Patient Educ Couns 99:1099-1105

22. Zomahoun HTV, Guenette L, Gregoire JP, Lauzier S, Lawani AM, Ferdynus C et al (2017) Effectiveness of motivational interviewing interventions on medication adherence in adults with chronic diseases: a systematic review and meta-analysis. Int J Epidemiol 46:589-602

23. Palacio A, Garay D, Langer B, Taylor J, Wood BA, Tamariz L (2016) Motivational interviewing improves medication adherence: a systematic review and meta-analysis. J Gen Intern Med 31:929-940

24. Graneheim UH, Lundman B (2004) Qualitative content analysis in nursing research: concepts, procedures and measures to achieve trustworthiness. Nurse Educ Today 24:105-112

25. Pereira-Salgado A, Westwood JA, Russell L, Ugalde A, Ortlepp B, Seymour JF et al (2017) Mobile health intervention to increase oral cancer therapy adherence in patients with chronic myeloid leukemia (The REMIND System): clinical feasibility and acceptability assessment. JMIR Mhealth Uhealth. 5:e184

26. Kelly TM, Daley DC, Douaihy AB (2012) Treatment of substance abusing patients with comorbid psychiatric disorders. Addict Behav 37:11-24

27. Lawrence DB, Allison W, Chen JC, Demand M (2008) Improving medication adherence with a targeted, technology-driven disease management intervention. Dis Manag 11:141-144

28. Verot E, RegnierDenois V, Macron C, Chauvin F (2020) Managing unresolved issues of addiction during cancer treatment: a qualitative study about cancer care providers' representations. PLoS One 15:e242693

29. Peddie N, Westbury T, Snowden A (2020) 'Nobody will put baby in the corner!': a qualitative evaluation of a physical activity intervention to improve mental health. Health Soc Care Community 28:2060-2075 
30. Lalla A, Salt S, Schrier E, Brown C, Curley C, Muskett O et al (2020) Qualitative evaluation of a community health representative program on patient experiences in Navajo Nation. BMC Health Serv Res 20:24

31. Huffman JC, Golden J, Massey CN, Feig EH, Chung WJ, Millstein RA et al (2021) A positive psychology-motivational interviewing program to promote physical activity in type 2 diabetes: The BEHOLD-16 pilot randomized trial. Gen Hosp Psychiatry 68:65-73

32. Myers B, Parry CDH, Morojele NK, Nkosi S, Shuper PA, Kekwaletswe CT et al (2020) "Moving forward with life": acceptability of a brief alcohol reduction intervention for people receiving antiretroviral therapy in South Africa. Int J Environ Res Public Health 17:5706

33. Miller WR, Rollnick S (1991) Motivational interviewing: preparing people to change addictive behavior. The Guilford Press, New York

34. Ryan RM, Deci EL (2000) Self-determination theory and the facilitation of intrinsic motivation, social development, and wellbeing. Am Psychol 55:68-78

35. Patrick H, Williams GC (2012) Self-determination theory: its application to health behavior and complementarity with motivational interviewing. Int J Behav Nutr Phys Act 9:18

36. Markland D, Ryan RM, Tobin VJ, Rollnick S (2005) Motivational interviewing and self-determination theory. J Soc Clin Psychol 24:811-831

37. Leow K, Lynch MF, Lee J (2021) Social support, basic psychological needs, and social well-being among older cancer survivors. Int J Aging Hum Dev 92:100-114

38. Yildiz E, Aylaz R (2020) How counseling based on acceptance and commitment therapy and supported with motivational interviewing affects the perceptions of treatment motivation in patients diagnosed with schizophrenia: a qualitative study. J Am Psychiatr Nurses Assoc. https://doi.org/10.1177/1078390320945380
39. Abughosh SM, Vadhariya A, Johnson ML, Essien EJ, Esse TW, Serna O et al (2019) Enhancing statin adherence using a motivational interviewing intervention and past adherence trajectories in patients with suboptimal adherence. J Manag Care Spec Pharm 25:1053-1062

40. Tzikas A, Samaras A, Kartas A, Vasdeki D, Fotos G, Dividis G et al (2020) Motivational Interviewing to Support Oral AntiCoagulation adherence in patients with non-valvular Atrial Fibrillation (MISOAC-AF): a randomised clinical trial. Eur Heart J Cardiovasc Pharmacother. https://doi.org/10.1093/ehjcvp/pvaa0 39

41. Shannon R, Donovan-Hall M, Bruton A (2017) Motivational interviewing in respiratory therapy: what do clinicians need to make it part of routine care? A qualitative study. PLoS One. 12:e0187335

42. Zimmerman BS, Seidman D, Berger N, Cascetta KP, Nezolosky M, Trlica K et al (2020) Patient perception of telehealth services for breast and gynecologic oncology care during the COVID-19 pandemic: a single center survey-based study. J Breast Cancer 23:542-552

43. Cox A, Lucas G, Marcu A, Piano M, Grosvenor W, Mold F et al (2017) Cancer survivors' experience with telehealth: a systematic review and thematic synthesis. J Med Internet Res 19:e11

44. Pham Q, Hearn J, Gao B, Brown I, Hamilton RJ, Berlin A et al (2020) Virtual care models for cancer survivorship. NPJ Digit Med. 3:113

Publisher's note Springer Nature remains neutral with regard to jurisdictional claims in published maps and institutional affiliations.

\section{Authors and Affiliations}

\section{Myriam Gagné ${ }^{1}(1)$. Sophie Lauzier ${ }^{2,3,4} \cdot$ Martine Lemay $^{5} \cdot$ Carmen G. Loiselle $^{6} \cdot$ Louise Provencher $^{7}$. Chantale Simard $^{8} \cdot$ Laurence Guillaumie $^{2,9,10}$ (1)}

Myriam Gagné

Myriam.Gagne@unityhealth.to

Sophie Lauzier

Sophie.Lauzier@crchudequebec.ulaval.ca

Martine Lemay

Martine.Lemay@chudequebec.ca

Carmen G. Loiselle

Carmen.g.Loiselle@mcgill.ca

Louise Provencher

Louise.Provencher@chudequebec.ca

Chantale Simard

Chantale1_Simard@uqac.ca

1 Unity Health Toronto (St. Michael's Hospital), affiliated with the University of Toronto, Toronto, ON, Canada

2 Population Health and Optimal Health Practices Research Axis, CHU de Québec Research Center-Université Laval, Québec City, QC, Canada
3 Faculty of Pharmacy, Université Laval, Québec City, QC, Canada

4 Équipe de recherche Michel-Sarrazin en oncologie psychosociale et soins palliatifs (ERMOS), Québec City, QC, Canada

5 Centre des maladies du sein, CHU de Québec-Université-Laval, Québec City, QC, Canada

6 Department of Oncology and Ingram School of Nursing, McGill University, Montreal, QC, Canada

7 Cancer Research Centre, Université Laval, Québec City, QC, Canada

8 Université du Québec à Chicoutimi, Chicoutimi, QC, Canada

9 Faculty of Nursing, Université Laval, Québec City, QC, Canada

10 Faculté des Sciences Infirmières, Université Laval Pavillon Ferdinand-Vandry, Local 3443, Québec, Québec G1V 0A6, Canada 